\title{
Enhancing Light Trapping of Macroporous Silicon by Alkaline Etching: Application for the Fabrication of Black Si Nanospike Arrays
}

\author{
G. Loget, ${ }^{a *}$ A. Vacher, ${ }^{a}$ B. Fabre, ${ }^{a}$ F. Gouttefangeas, ${ }^{b}$ L. Joanny ${ }^{b}$ and V. Dorcet ${ }^{c}$
}

\begin{abstract}
a. Institut des Sciences Chimiques de Rennes, UMR 6226 CNRS, Matière Condensée et Systèmes Electroactifs (MaCSE), Université de Rennes 1, Campus de Beaulieu, 35042 Rennes Cedex, France. b. ScanMAT-Cmeba, Université de Rennes 1, Campus de Beaulieu, 35042 Rennes Cedex, France. c. Pôle Ressources Administratives Techniques et Scientifiques (PRATS), Université de Rennes 1, Campus de Beaulieu, 35042 Rennes Cedex, France.
\end{abstract}

Email : gabriel.loget@univ-rennes1.fr

ABSTRACT: The synthesis of highly absorbing silicon (black Si (BSi)) is a very active research topic with promising applications in the field of sustainable energies, ultrasensitive sensing and antibacterial materials. Here, we show that extended alkaline etching of macroporous $\mathrm{Si}$, fabricated by photoelectrochemical etching drastically influence the surface structures as well as their optical properties. We demonstrate that this treatment can considerably improve the light trapping of the surface and we finally show that it is possible to use it for fabricating very quickly highly-absorbing arrays of sharp and crystalline BSi nanospikes (NSpikes).

\section{INTRODUCTION}

The high reflection of light by silicon surfaces is a critical issue for many Si-based devices as it limits electron-hole pair generation and therefore restrains the performances of Si-based energy conversion devices such as solar cells (SCs), water-splitting photoelectrochemical cells (PECs) and photodetectors. ${ }^{1-5}$ It is known that enhanced light absorption can arise from nano- and microstructured Si surfaces that induce respectively a smooth transition of refractive index or multiple internal reflections. ${ }^{1,4,6,7}$ In this context, top down micro- and nanostructuration of mono- or polycrystalline Si surfaces is highly appealing for boosting photon absorption and enlarging surface areas while conserving the properties of bulk $\mathrm{Si}$. The fabrication of structured and highly absorbing Si, referred as Black $\mathrm{Si}(\mathrm{BSi})$, is, therefore, a very active research topic in materials with applications for renewable energy and photonics ${ }^{1-4,8-10}$ and also, as recently demonstrated, for designing efficient surface-enhanced Raman scattering (SERS) ${ }^{11}$ surfaces or biomimetic antibacterial materials ${ }^{12}$. Several techniques can be used to enhance light absorption. Dry etching processes such as reactive ion 
etching (RIE) or deep RIE are usually employed to fabricate grass-like nanotip arrays with broadband low reflectance. ${ }^{13-15}$ In addition, the group of Mazur reported that irradiation of Si wafer with femtosecond-laser pulses in the presence of a halogen or a fluoride-containing gas can be used to create random arrays of absorbing nanocones. ${ }^{16}$ If these dry techniques are highly tunable, their main drawbacks are their cost and the handling of gases. Conversely, wet chemical approaches usually require simple experimental setups and hold fast etching rates which make them highly promising for the large-scale production of $\mathrm{BSi}^{2}{ }^{2}$ Among them, alkaline etching (AE) of Si by $\mathrm{KOH}^{17}$ or tetramethylammonium hydroxide solutions, ${ }^{18,19}$ is largely used for structuring solar cells and for manufacturing microelectromechanical systems. While these processes can be used to create random arrays of Si micropyramids, the resulting weighted reflectivity is usually limited. ${ }^{20,21}$ Stain etching can also be used to produce an antireflective porous layer, ${ }^{22,23}$ however, the reproducibility of this process is often discussed. ${ }^{2,24}$ Metal-assisted chemical etching is a very popular method for manufacturing absorbing Si nanowires (SiNWs) ${ }^{25}$ and porous BSi that can improve the performances of SCs, ${ }^{8}$ however this process can contaminate the surface with metals. ${ }^{2}$ Besides, electrochemical methods are generally low-cost and allow a high degree of tunability. For instance, it has been reported that the electroreduction of silica ${ }^{26}$ can lead to $\mathrm{BSi}^{27}$ Electrochemical etching $(\mathrm{EE})^{28,29}$ and photoelectrochemical etching $(\mathrm{PEE})^{30-33}$ of $\mathrm{Si}$ are cost-effective and easy to implement methods that are intensely used to grow micropores (pores width $<2 \mathrm{~nm}$ ), mesopores $(2 \mathrm{~nm}<$ pores width $<50$ $\mathrm{nm}$ ) as well as random or organized arrays of macropores (pores width $>50 \mathrm{~nm}$ ) on mono- and polycrystalline $\mathrm{Si}^{24,34-36}$ In particular, great developments have been done on PEE of $n$-type Si illuminated from the backside by Lehman and Föll who developed the "space charge region pore formation model" and used it to fabricate well controlled Si macropore arrays. ${ }^{30,34}$ Levy-Clement et al. have reported the formation of macropores on frontside illuminated $n$-type $\mathrm{Si}^{3}, 3,37$ however much less work have been devoted to this structuration process. ${ }^{34} \mathrm{EE}$ and PEE can be used to produce $\mathrm{BSi}$, notably, it has been reported that thin layers of micro- and mesoporous Si can lead to a substantial reflectivity decrease. ${ }^{38}$ In addition, thicker macropore layers can also confer antireflective properties to $\mathrm{Si}^{39-42}$ Interestingly, our group and others have recently shown that BSi made of organized or random macropores fabricated using PEE exhibit enhanced performance as photoanodes for water splitting PECs. ${ }^{40,41}$ During typical syntheses of macroporous $\mathrm{Si}$, a short exposure of PEE-etched porous $\mathrm{Si}$, in weakly concentrated $\mathrm{KOH}$ at ambient temperature is commonly employed to smoothen the pore walls. ${ }^{30,33,36}$ However, the effect of this treatment on porous Si seems to have been overlooked and, to the best of our knowledge, the extended etching of porous Si by highly concentrated and hot alkaline solutions have never been studied so far. In this work, we show that this treatment drastically influences the surface structures as well as their optical properties. We demonstrate that 
it can considerably improve the light trapping of the surfaces and we finally use this effect to produce very quickly ( $\sim \mathrm{min}$ ) BSi made of Si NSpikes on large areas.

\section{EXPERIMENTAL SECTION}

Materials and Reagents. Acetone (MOS electronic grade, Erbatron from Carlo Erba) and anhydrous ethanol (RSE electronic grade, Erbatron from Carlo Erba) were used without further purification for cleaning the silicon surfaces. The ultrapure water had a resistivity of $18.2 \mathrm{M} \Omega \mathrm{cm}$ (Purelab Classic UV from Veolia Water STI). HF (50\% aq. solutions), and $\mathrm{KOH}$ (> $85 \%$, Reag. Ph. Eur.) were purchased from Sigma-Aldrich. The $n$-type silicon wafers (1-5 W cm resistivity, phosphorus doped, double side polished, 250-275 mm thickness, (100)) were purchased from Siltronix. The chemicals used for the cleaning and etching of the Teflon vials $\left(30 \% \mathrm{H}_{2} \mathrm{O}_{2}\right.$ and $\left.96-97 \% \mathrm{H}_{2} \mathrm{SO}_{4}\right)$ were of $\mathrm{VLSI}\left(\mathrm{H}_{2} \mathrm{O}_{2}\right.$, from Sigma-Aldrich) and MOS ( $\mathrm{H}_{2} \mathrm{SO}_{4}$ from BASF) semiconductor grade. The absolute ethanol used for PEE and isopropanol were purchased from VWR.

Photoelectrochemical Etching. All Teflon vials and tweezers used for cleaning of silicon were previously decontaminated in $3 / 1 \mathrm{v} / \mathrm{v}$ concentrated $\mathrm{H}_{2} \mathrm{SO}_{4} / 30 \% \mathrm{H}_{2} \mathrm{O}_{2}$ at $100{ }^{\circ} \mathrm{C}$ for $30 \mathrm{~min}$, followed by copious rinsing with ultrapure water. Caution: the concentrated aqueous $\mathrm{H}_{2} \mathrm{SO}_{4} / \mathrm{H}_{2} \mathrm{O}_{2}$ (piranha) solution is very dangerous, particularly in contact with organic materials, and should be handled extremely carefully. The $n$-type silicon (100) wafers were cut in $1.5 \times 1.5 \mathrm{~cm}^{2}$ squares and degreased by sonication (10 $\mathrm{min}$ ) in acetone, ethanol and ultrapure water in Teflon vials. They were then dried under an Ar stream. One side of the Si surface was scratched using a diamond glass cutter and sandpaper to create trenches in the native oxide layer. For the photoelectrochemical etching, the surfaces were inserted into a home-made open-top O-ring cell (the diameter of the O-ring was $1 \mathrm{~cm}$ ). The electrical contact was achieved by pressing an aluminum foil between the scratched Si surface and a copper plate. The counter electrode was a Pt ring $(1 \mathrm{~cm}$ diameter) placed above the silicon surface. The cell was filled with $4 \mathrm{~mL}$ of the electrolyte solution containing absolute ethanol and $50 \%$ aq. $\mathrm{HF}$ at a $1 / 1 \mathrm{v} / \mathrm{v}$ ratio. The Si surface was irradiated from the electrolyte side with a solar simulator (LS0106, LOT Quantum Design) equipped with an AM 1.5G filter and a current of $100 \mathrm{~mA}$ was applied for 4 min with a LAB-SMP (ET System) power supply. During the PEE, the current flowing through the cell was monitored using a Keithley 2000 and recorded on a computer via a homemade Labview program. The surface was then copiously rinsed with absolute ethanol and dried under an argon flow. 
Alkaline Etching. The AE solution was freshly prepared by dissolving $\mathrm{KOH}\left(500 \mathrm{~g} \mathrm{~L}^{-1}, 8.9 \mathrm{M}\right)$ in a water/isopropanol ( $2 \%$ vol.) mixture. This solution was maintained at $80^{\circ} \mathrm{C}$ and vigorously stirred (350 rpm) in a Pyrex beaker. After PEE, the ethanol-rinsed and dried porous Si surfaces were directly exposed to the AE solution for the desired amount of time (between $2 \mathrm{~s}$ and $2 \mathrm{~min}$ ). After that, they were dipped in ultrapure water to stop the etching, rinsed copiously and dried with Ar.

Surface Characterization. Scanning electron microscopy (SEM) was performed using a JSM 7100F (JEOL). SEM image analysis was performed using the ImageJ software. Energy-dispersive X-ray spectroscopy was done using a Silicon Drift Detector (SDD) - X-Max $\left(80 \mathrm{~mm}^{2}\right)$ and the AZtecEnergy software (Oxford Instruments). Transmission electron microscopy (TEM) was performed with a JEOL $2100 \mathrm{LaB}_{6}$ microscope operating at 200kV equipped with a GATAN Orius 200D CCD camera for diffraction and Bright Field (BF) images acquisition and with a GATAN UItra-Scan 1000 CCD camera for high-resolution images acquisition. The reflectance spectra were acquired on a Shimadzu UV3600Plus spectrophotometer with an integrating sphere (ISR-603). A sample of extra pure $\mathrm{BaSO}_{4}$ was used as the $100 \%$ reflectance reference (Nacalai Tesque). A Teflon mask was used to perform the measurement and ensure a good reproducibility. The total reflectance was measured with an incident beam with an angle of $8^{\circ}$ to include the specular component.

\section{RESULTS AND DISCUSSION}

PEE was performed with a two-electrode setup, in the photoelectrochemical cell depicted in the inset of Figure $1 \mathrm{~b}$ with a classical ethanol-based electrolyte containing HF. ${ }^{24}$ We employed light irradiation to supply holes for oxidizing the $n$-type Si surface, which was illuminated from the top of the cell (from the electrolyte side). It is worth mentioning that this illumination configuration, as well as the spectral distribution of the incident light (AM 1.5G), have an importance on the pore morphology. The PEE parameters were kept constant for all the samples made in this work: a current density $j=$ $0.13 \mathrm{~A} \mathrm{~cm}^{-2}$ was applied for $4 \mathrm{~min}$ in every case. As shown in Figure S1, the potential typically stabilized at $\sim 20 \mathrm{~V}$ after $40 \mathrm{~s}$. In these conditions, the combined action of photogenerated holes and fluorides in solution led to the dissolution of the Si surface and the formation of a porous Si (PSi) layer, as described by the following reaction: ${ }^{24,30}$

$$
\mathrm{Si}_{(\mathrm{s})}+6 \mathrm{HF}_{(\mathrm{aq})}+2 \mathrm{~h}^{+} \rightarrow \mathrm{SiF}_{6}{ }^{2-}{ }_{(\mathrm{aq})}+\mathrm{H}_{2(\mathrm{~g})}+4 \mathrm{H}^{+}{ }_{(\mathrm{aq})}
$$


The pores first nucleated randomly on the surface and propagated along the [100] direction because of the combined action of the current flowing preferentially at the bottom of the pores, the passivation of the pore walls and the preferential etching of the (100) faces. ${ }^{30,43}$ The so-patterned layers were then characterized by SEM. As shown in Figure $1 \mathrm{~b}$ and S2, the surfaces after PEE comprised a $60 \mathrm{~mm}$-thick porous layer composed of $\sim 50 \mathrm{~mm}$-thick straight macropores aligned in the [100] direction and spaced by 1-2 mm, these pores being covered by a $10 \mathrm{~mm}$-thick PSi crust, referred as the transitional layer, that is known to be generated during the nucleation of the pores. ${ }^{35}$ A closer investigation of the top-crust (see Figure S2b) and the inner cavity of the macropores, shown in Figure 1c, revealed that they were both filled with a micro-/mesoporous layer, as previously reported in other works employing PEE of similarly doped $n$-Si with a frontside illumination configuration. ${ }^{24,30,31}$ After PEE, the PSi surfaces were directly immersed in a hot $\left(80^{\circ} \mathrm{C}\right)$ solution of 8.9 $\mathrm{M} \mathrm{KOH}$ to which has been added $2 \%$ vol. of isopropanol, in order to improve the wetting of the hydrophobic porous layer. Upon exposure to the $\mathrm{KOH}$ solution, Si spontaneously reacted following: ${ }^{35}$

$$
\mathrm{Si}_{(\mathrm{s})}+2 \mathrm{OH}^{-}{ }_{(\mathrm{aq})}+2 \mathrm{H}_{2} \mathrm{O}_{(\mathrm{aq})} \rightarrow \mathrm{SiO}_{2}(\mathrm{OH})_{2}{ }^{2-}{ }_{(\mathrm{aq})}+2 \mathrm{H}_{2(\mathrm{~g})}
$$

A vigorous bubble evolution, corresponding to the generation of $\mathrm{H}_{2}$ at the Si surface was observed, confirming that reaction (2) was occurring at a high rate. In order to efficiently remove the bubbles from the $\mathrm{Si}$, the solution was constantly stirred at high speed. The $\mathrm{H}_{2}$ evolution was especially high during the first seconds of etching and continued at a slower rate over the whole duration of our experiments (up to $2 \mathrm{~min}$ ). A series of samples was prepared by using several PSi samples (all prepared by the PEE protocol previously described) and exposing them for different times to the $\mathrm{KOH}$ solution in order to study the effect of reaction (2) on the morphology of the Si surface as a function of time. The SEM images of samples obtained during the first seconds of reaction are shown in Figure S3 and S4 (etched for $2 \mathrm{~s}$ and $15 \mathrm{~s}$, respectively) and samples exposed to the AE solution from $30 \mathrm{~s}$ to $2 \mathrm{~min}$, are shown in Figure 2.

Due to the enormous surface area of micro-/mesoporous Si (typically in the order of 100 to $1000 \mathrm{~m}^{2}$ $\left.\mathrm{cm}^{-3}\right),{ }^{44}$ this material reacted at a much higher rate to the alkaline solution than the bulk Si. Consequently, the PSi crust that was observed on the top of the surface after PEE (Figure 1b) dissolved quasi-instantaneously. Figure S3 shows that this top layer disappeared after $2 \mathrm{~s}$ of reaction. However, a close investigation of the macropore walls for surfaces that were exposed for $2 \mathrm{~s}$ and $15 \mathrm{~s}$ (Figure S3d and S4d) revealed that a micro-/mesoporous layer was still present inside the macropores. We attribute this to the lack of hydroxide inside the pores during the first seconds of $A E$, caused by the limited accessibility within these deep structures. After $30 \mathrm{~s}$ of reaction, only bulk, macroporous Si could be observed, suggesting that all micro-/mesoporous Si was etched. 

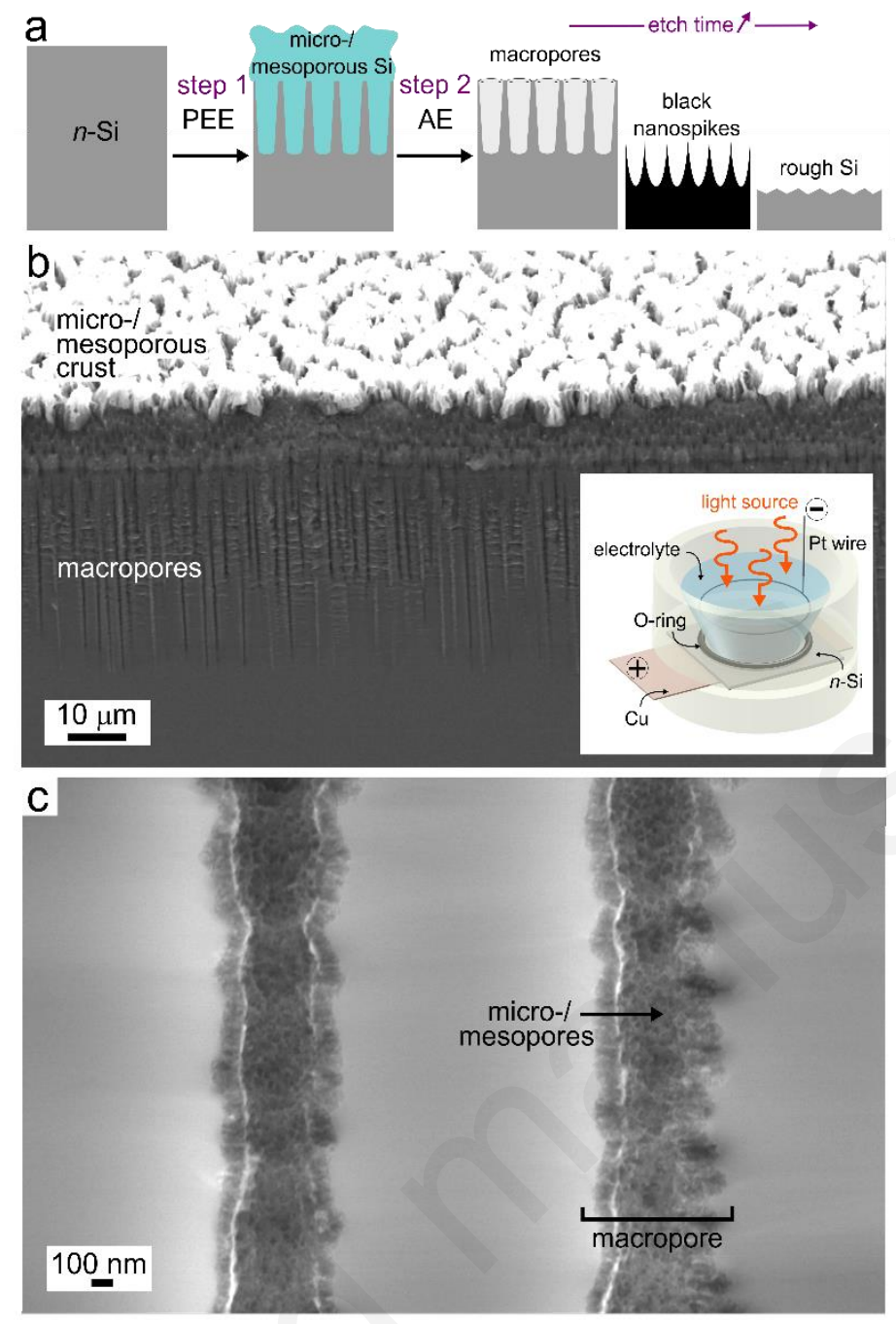

Figure 1. a) Two-step structuration procedure involving a first photoelectrochemical etching (PEE) followed by an alkaline etching (AE). b) Low magnification tilted view $\left(45^{\circ}\right)$ showing the macropores covered by a micro-/mesoporous transitional layer (Si layer obtained after a PEE of 4 min at $0.13 \mathrm{~A} \mathrm{~cm}^{-}$ ${ }^{2}$ ). Inset: Scheme of the photoelectrochemical setup used for PEE. c) High magnification cross-section showing the inner walls of two macropores.

Although SEM reveals that the micro-/mesoporous Si was totally dissolved after $30 \mathrm{~s}$, the surface continuously reacted with the hydroxides and the macropores morphology consequently evolved during the exposure to the $\mathrm{KOH}$ solution, as shown in Figure 2. The tilted and cross-section SEM views clearly show the evolution of the micro- and nanostructures as reaction (2) occurs (Figure S6, which presents the layer thickness as a function of etching time, reveals an etching rate of $320 \mathrm{~nm} \mathrm{~s}^{-}$ $\left.{ }^{1}\right)$. Figure 2 shows that at $30 \mathrm{~s}$ of $\mathrm{AE}$, the structures could be considered conical macropores. As it can be seen in the SEM top views (in the bottom row of Figure 2), the thickness of the pore walls was not homogeneous, therefore some sections of the walls dissolved at a slower rate than others. This is 
particularly true for the walls that were shared by several macropores (typically $>3-4$ ), some of them are indicated by the white circles in the SEM top views (bottom row of Figure 2). As the macropores opened, it can be observed that Si NSpikes started to develop in these specific areas, which were initially thicker that the rest of the wall. This was caused by a faster etching of the top of the pore walls than the bottom, which we attribute to the gradual decrease of the hydroxide concentration along the pores, caused by their low diffusion within the cavities. The formation of these NSpikes can be observed in the cross-section images obtained from $45 \mathrm{~s}$ and they are particularly pronounced for the samples immersed in the AE solution for 1 and $1.5 \mathrm{~min}$. At $1.5 \mathrm{~min}$, the pores totally disappeared due to the full dissolution of their walls and the surface was only composed of $5 \mathrm{~mm}$ long NSpikes, spaced by inverted pyramids pits (with exposed (111) faces), resulting from the anisotropic AE of the macropore bottoms. ${ }^{17-19}$ Ultimately, when the reaction was carried out for extended times (here 2 min, as shown in the last column of Figure 2), the NSpikes were totally etched and only a rough $\mathrm{Si}$ surface remained. We observed an intriguing color change of the surface occurring during the $A E$ reaction, and have consequently investigated the optical properties of the structured surfaces. In order to simply visualize the color changes as a function of the AE time, several samples were prepared with different AE times, and their photographs are displayed in Figure S7. The total reflectance as a function of the reaction time was also measured and the corresponding spectra are shown in Figure 3. The reflectance of planar (100) Si was comprised between $50 \%$ and $30 \%$, with an average total reflectance of $37 \%$ in the visible range $(400-800 \mathrm{~nm})$, which is in good agreement with previous reports. ${ }^{2}$ After PEE, the surface became mainly bright yellow. We attribute this color to the high scattering of light by the micro-/mesoporous Si crust present on the top of the macropores (Figure S2). We can state from Figure 3 that the AE was divided into two periods of time.

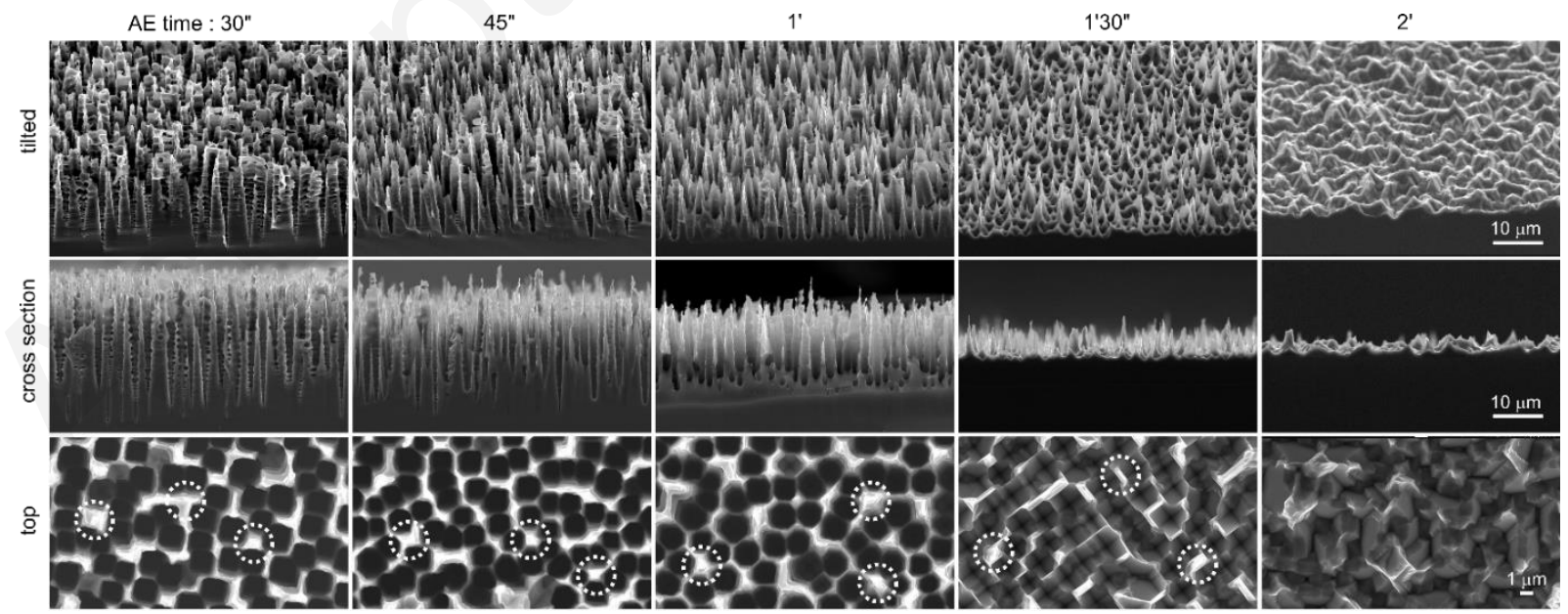

Figure 2. SEM images showing the Si porous layer obtained after different times of $A E$ (note that in all cases, the surface was patterned with an identical PEE step of $4 \mathrm{~min}$ at $0.13 \mathrm{~A} \mathrm{~cm}^{-2}$ ). The time of AE is indicated on the top of each column. Top, middle and bottom rows show tilted $\left(45^{\circ}\right)$, cross-section and 
top views, respectively (For sake of visibility, three areas where a NSpike forms are indicated by white dotted circles in the four first images of the bottom row).

As the macropores opened, it can be observed that Si NSpikes started to develop in these specific areas, which were initially thicker that the rest of the wall. This was caused by a faster etching of the top of the pore walls than the bottom, which we attribute to the gradual decrease of the hydroxide concentration along the pores, caused by their low diffusion within the cavities. The formation of these NSpikes can be observed in the cross-section images obtained from $45 \mathrm{~s}$ and they are particularly pronounced for the samples immersed in the AE solution for 1 and $1.5 \mathrm{~min}$. At $1.5 \mathrm{~min}$, the pores totally disappeared due to the full dissolution of their walls and the surface was only composed of $5 \mathrm{~mm}$ long NSpikes, spaced by inverted pyramids pits (with exposed (111) faces), resulting from the anisotropic $A E$ of the macropore bottoms. ${ }^{17-19}$ Ultimately, when the reaction was carried out for extended times (here $2 \mathrm{~min}$, as shown in the last column of Figure 2), the NSpikes were totally etched and only a rough Si surface remained. We observed an intriguing color change of the surface occurring during the $\mathrm{AE}$ reaction, and have consequently investigated the optical properties of the structured surfaces. In order to simply visualize the color changes as a function of the AE time, several samples were prepared with different AE times, and their photographs are displayed in Figure S7. The total reflectance as a function of the reaction time was also measured and the corresponding spectra are shown in Figure 3. The reflectance of planar (100) Si was comprised between $50 \%$ and $30 \%$, with an average total reflectance of $37 \%$ in the visible range (400-800 $\mathrm{nm}$ ), which is in good agreement with previous reports. ${ }^{2}$ After PEE, the surface became mainly bright yellow. We attribute this color to the high scattering of light by the micro-/mesoporous Si crust present on the top of the macropores (Figure S2). We can state from Figure 3 that the AE was divided into two periods of time. During the first stage, i.e. for AE times comprised between $2 \mathrm{~s}$ and $1 \mathrm{~min}$, the surface reflectance continuously decreased, showing that the light trapping was considerably enhanced by the opening of the macropores and the growth of the Si NSpikes (Figure 2) that allowed for a better light penetration in these high-aspect-ratio structures and favored multiple internal reflections. As shown in Figure 3b, the optimal "blackness", i.e. the lower reflectance, was obtained for $A E$ times of $45 \mathrm{~s}$ and $1 \mathrm{~min}$, corresponding to the surfaces bearing the sharp and high aspect ratio Si NSpike arrays. During the second stage of AE (after $1 \mathrm{~min}$ ), the surface reflectance increased, due to the lowering of the aspect ratio of the nanostructures, as shown in Figure 2. The average total reflectance for the best light trapping surfaces was $3 \%$ in the visible range, meaning that this treatment induced a reflectance decrease of $90 \%$ with respect to the polished planar Si. Additional optical measurements, performed with another spectrophotometer were in very good agreement 
(Figure S8). This reflectance is much lower than that of our previously reported macroporous $\mathrm{Si}^{41}$ and the typical values obtained for the widely used micropyramid arrays. ${ }^{18-21}$ This value is rather in the same range as those reported for Si surfaces structured by femtosecond laser pulses, ${ }^{16}$ modified with a porosity gradient ${ }^{38}$ or with regular arrays of Si modulated macropores, ${ }^{39,40}$ and our process is simpler. These results show that: i) AE can have a drastic effect on the shape of macroporous Si and can result in totally new nanostructures and ii) the so-fabricated structures can highly enhance the light absorption.
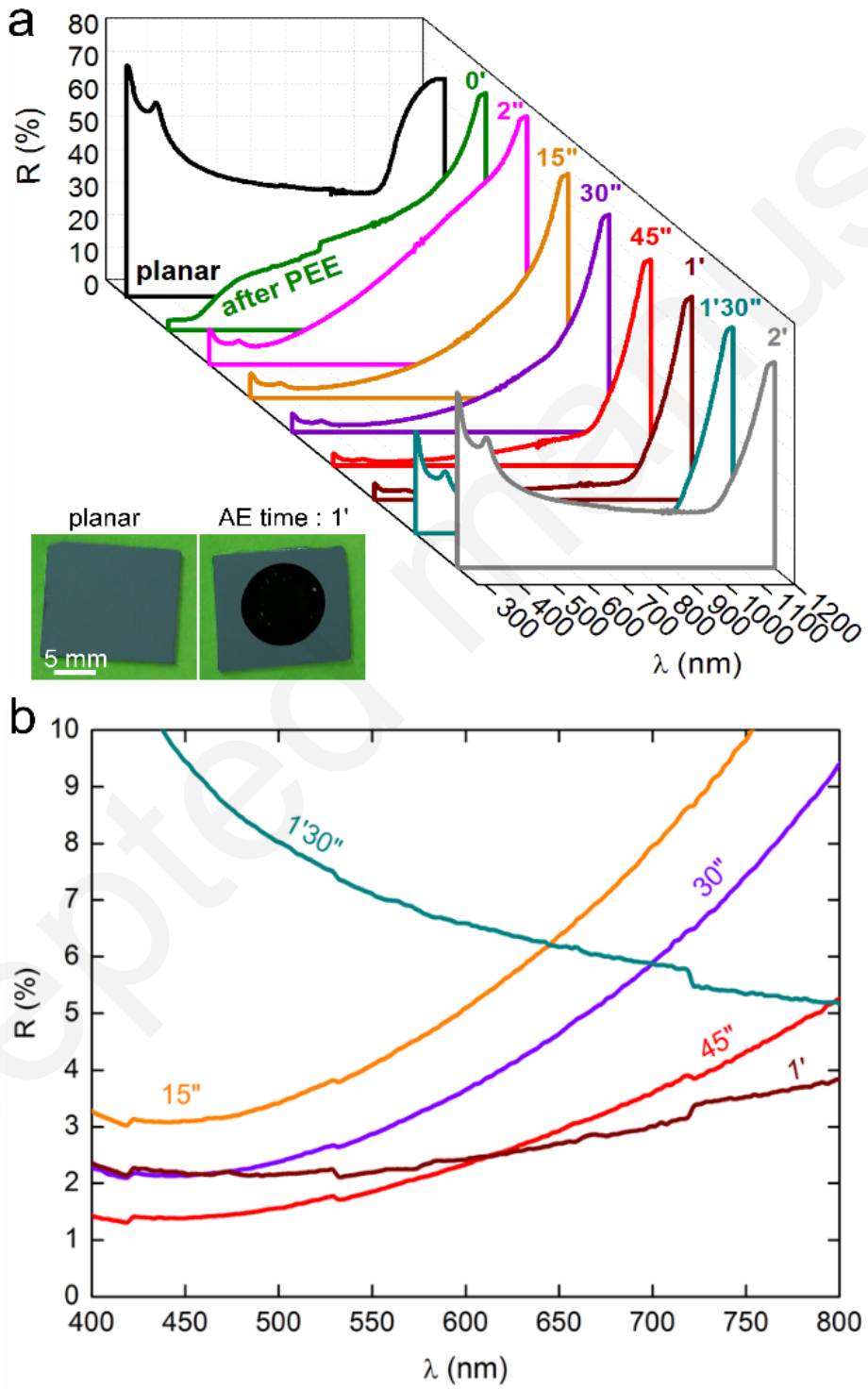

Figure 3. a) Series of UV-vis-NIR total reflectance spectra showing the evolution of the reflectance as a function of the AE time. Inset: Photograph of a planar Si surface (left) and a Si NSpike surface (right). b) Reflectance in the visible range for the most absorbing samples. 


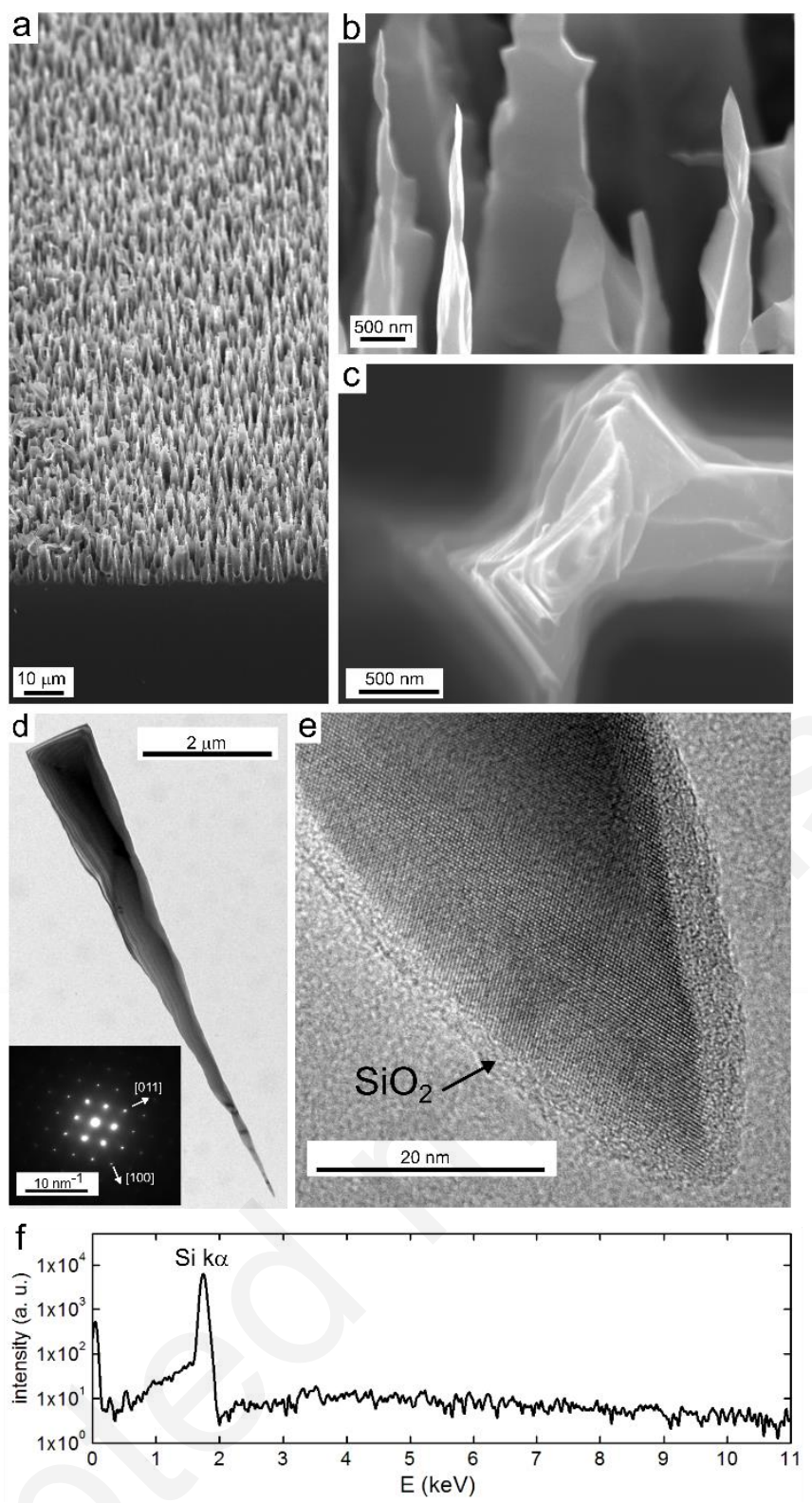

Figure 4. a-c) SEM images showing: a) a low magnification tilted view $\left(45^{\circ}\right)$, b) a high magnification tilted view and $c$ ) a high magnification top view of a NSpike surface that was obtained for an AE time of 1 min. d) Bright-field TEM image of an individual Si NSpike detached from a Si surface that underwent AE for $1 \mathrm{~min}$. Inset: Diffraction pattern obtained in the middle of the Si NSpike. e) Highresolution TEM image showing the tip of the corresponding Si NSpike. f) EDS spectrum of the corresponding surface (note that the $y$ - axis is in logarithmic scale).

We now pay special attention to the Si NSpike arrays that presented the best light trapping because they are very promising for energy conversion, SERS or biomimetic antibacterial devices. ${ }^{10-12}$ More samples were prepared using the protocol described above (with an AE time of $1 \mathrm{~min}$ ) and were further characterized, as shown in Figure 4. In the low magnification view (Figure 4a), it can be seen 
that the NSpikes covered the whole surface of the Si wafer with a grass-like fashion (see also Figure S9), based on analyses of SEM top views, the surface density was estimated to be $7 \times 10^{4} \mathrm{~mm}^{-2}$. Interestingly, the shape of the so-fabricated NSpikes was closely similar to that of structures obtained by $\mathrm{RIE}^{15}$ although their length was, in our case, at least one order of magnitude greater. High magnification SEM images, shown in Figure $4 b$ and $4 c$ reveal that the tips of the spikes were sharp and highly faceted. The Si NSpikes were further investigated by transmission electron microscopy (TEM), which allowed to observe individual Si NSpikes (Figure 4d) detached from the Si surface by scratching. TEM images, shown in Figure $4 \mathrm{~d}$ and Figure $\mathrm{S} 10$ as well as the diffraction pattern in the inset of Figure $4 d$, reveal that the Si NSpikes were monocrystalline and confirmed their orientation in the [100] direction. Interestingly, the high-resolution (HR) TEM observations presented in Figure 4e and Figure S10 confirm that the NSpikes apexes were in the range of $10 \mathrm{~nm}$ and showed that the $\mathrm{Si}$ NSpikes were covered by a $\sim 3 \mathrm{~nm}$-thick native amorphous $\mathrm{SiO}_{2}$ layer. EDS analyses of the NSpike arrays, presented in Figure $4 \mathrm{f}$ for a large area (see Figure S11 for the analysis area), and in Figure S12 for an individual Si NSpike both show that Si was the only detected element, which demonstrates that the surfaces were free of potassium or fluorine contaminants that may have an undesired influence on the Si properties.

\section{CONCLUSIONS}

In summary, we have reported the effect of extended AE on macroporous silicon. We have shown that this surface treatment can lead to new nanostructures that have a significant effect on the light trapping of the surface. We have shown by SEM, TEM, and optical measurements that reaction optimization allows the generation of arrays of sharp, crystalline and highly absorbing crystalline $\mathrm{Si}$ NSpikes presenting an average reflectance of $3 \%$ in the visible range. Due to the potential scalability and the simplicity of the method, it is highly promising because such arrays could have straightforward applications for various Si-based devices, in particular for improving the performances of $\mathrm{SCs},{ }^{5}$ as well as in artificial photosynthesis for producing highly efficient Si-based PECs photoelectrodes. ${ }^{1}$ Last but not least, such spikes would be appealing for ultrasensitive biodetection devices based on surface-enhanced Raman scattering (SERS) ${ }^{11}$ or for designing antibacterial materials. ${ }^{12}$ 


\section{ACKNOWLEDGMENTS}

This work is funded by ANR (project EASi-NANO, ANR-16-CE09-0001-01). Thierry Guizouarn is acknowledged for making the labview program used for electrochemical etching. Dr. F. Cheviré is acknowledged for discussion about the spectrophotometer and reflectance measurements. The authors acknowledge the THEMIS platform for TEM facilities.

\section{REFERENCES}

1 K. Sun, S. Shen, Y. Liang, P. E. Burrows, S. S. Mao and D. Wang, Chem. Rev., 2014, 114, 86628719.

2 X. Liu, P. R. Coxon, M. Peters, B. Hoex, M. Cole and D. J. Fray, Energy Environ. Sci., 2014, 7, 3223-3263.

3 J. E. Carey, C. H. Crouch, M. Shen and E. Mazur, Opt. Lett., 2005, 30, 1773-1775.

4 F. Priolo, T. Gregorkiewicz, M. Galli and T. F. Krauss, Nat. Nanotech., 2014, 9, 19-32.

5 J. Zhu, Z. Yu, S. Fan and Y. Cui, Mater. Sci. Eng. R-Rep., 2010, 70, 330-340.

6 S. Lou, X. Guo, T. Fan and D. Zhang, Energy Environ. Sci., 2012, 5, 9195-9216.

7 M. Toma, G. Loget and R. M. Corn, Nano Lett., 2013, 13, 6164-6169.

8 J. Oh, H.-C. Yuan and H. M. Branz, Nat. Nanotechnol., 2012, 7, 743-748.

9 M. Otto, M. Kroll, T. Käsebier, S.-M. Lee, M. Putkonen, R. Salzer, P. T. Miclea and R. B. Wehrspohn, Adv. Mater., 2010, 22, 5035-5038.

10 M. Otto, M. Algasinger, H. Branz, B. Gesemann, T. Gimpel, K. Füchsel, T. Käsebier, S. Kontermann, S. Koynov, X. Li, V. Naumann, J. Oh, A. N. Sprafke, J. Ziegler, M. Zilk and R. B. Wehrspohn, Adv. Opt. Mater., 2014, 1-18.

11 Y. Chen, G. Kang, A. Shah, V. Pale, Y. Tian, Z. Sun, I. Tittonen, S. Honkanen and H. Lipsanen, Adv. Mater. Interfaces, 2014, 1, 1300008.

12 E. P. Ivanova, J. Hasan, H. K. Webb, G. Gervinskas, S. Juodkazis, V. K. Truong, A. H. F. Wu, R. N. Lamb, V. A. Baulin, G. S. Watson, J. A. Watson, D. E. Mainwaring and R. J. Crawford, Nat. Commun., 2013, 4, 2838.

13 C. Lee, S. Y. Bae, S. Mobasser and H. Manohara, Nano Lett., 2005, 5, 2438-2442.

14 J. Zhu, Z. Yu, G. F. Burkhard, C. Hsu, S. T. Connor, Y. Xu, Q. Wang, M. Mcgehee, S. Fan and Y. C ui, Nano Lett., 2009, 9, 279-282.

15 Y.-F. Huang, S. Chattopadhyay, Y.-J. Jen, C.-Y. Peng, T.-A. Liu, Y.-K. Hsu, C.-L. Pan, H.-C. Lo, C.H. Hsu, Y.-H. Chang, C.-S. Lee, K.-H. Chen and L.-C. Chen, Nat. Nanotechnol., 2007, 2, 770-774. 

and A. Karger, Appl. Phys. Lett., 2001, 78, 1850-1852.

E. D. Palik, O. J. Glembocki, I. J. Heard, P. S. Burno and L. Tenerz, J. Appl. Phys., 1991, 70, 3291-3300.

D. Iencinella, E. Centurioni, R. Rizzoli and F. Zignani, Sol. Energy Mater. Sol. Cells, 2005, 87, 725-732.

P. Papet, O. Nichiporuk, A. Kaminski, Y. Rozier, J. Kraiem, J.-F. Lelievre, A. Chaumartin, A. Fave and M. Lemiti, Sol. Energy Mater. Sol. Cells, 2006, 90, 2319-2328.

J. Xiao, L. Wang, X. Li, X. Pi and D. Yang, Appl. Surf. Sci., 2010, 257, 472-475.

21

T.-G. Truong, C. Meriadec, B. Fabre, J.-F. Bergamini, O. de Sagazan, S. Ababou-Girard and G. Loget, Nanoscale, 2017, 9, 1799-1804.

M. T. Kelly, J. K. M. Chun and A. B. Bocarsly, Appl. Phys. Lett., 1994, 64, 1693-1695.

R. Bilyalov, L. Stalmans and J. Poortmans, J. Electrochem. Soc. , 2003, 150, G216-G222.

M. J. Sailor, Porous Silicon in Practice, WILEY-VCH Verlag, 2012.

Z. Huang, N. Geyer, P. Werner, J. De Boor and U. Gösele, Adv. Mater., 2011, 285-308.

T. Nohira, K. Yasuda and Y. Ito, Nat. Mater., 2003, 2, 397-401.

E. Juzeliunas, A. Cox and D. J. Fray, Electrochim. Acta, 2012, 68, 123-127.

V. Lehmann and S. Ronnebeck, J. Electrochem. Soc. , 1999, 146, 2968-2975.

C. Lévy-Clément, S. Lust, S. Bastide, Q. N. Lê and D. Sarti, Phys. Status Solidi (a), 2003, 197, 27-33.

V. Lehmann and H. Föll, J. Electrochem. Soc. , 1990, 137, 653-659.

C. Lévy-Clément, A. Lagoubi, D. Ballutaud, F. Ozanam, J.-N. Chazalviel and M. NeumannSpallart, Appl. Surf. Sci., 1993, 65, 408-414.

C. Cozzi, G. Polito, K. W. Kolasinski and G. Barillaro, Adv. Funct. Mater., 2017, 27, 1604310.

C. Levy-Clement, A. Lagoubi, R. Tennes and M. Neumann-Spallart, Electrochim. Acta, 1992, $31,877-888$.

H. Föll, M. Christophersen, J. Carstensen and G. Hasse, Mater. Sci. Eng. R-Rep., 2002, 39, 93141.

35 X. G. Zhang, Electrochemistry of Silicon and Its Oxide, Kluwer Academic, 2001.

A. Langner, F. Müller and U. Gösele, in Molecular- and Nano-tubes, Speinger., 2011, pp. 431460. 4140. 
39 W. Huang, Y. Xue, X. Wang and X. Ao, Opt. Mater. Express, 2015, 5, 1082-1087.

40 X. Ao, X. Tong, D. S. Kim, L. Zhang, M. Knez, S. He and V. Schmidt, Appl. Phys. Lett., 2015, 111901.

41 L. Santinacci, M. W. Diouf, M. K. S. Barr, B. Fabre, L. Joanny, F. Gouttefangeas and G. Loget, ACS Appl. Mater. Interfaces, 2016, 8, 24810-24818.

42 J. Schilling, S. Matthias, R. B. Wehrspohn and U. Gösele, App. Phys. Lett., 2001, 1180, 2-5.

43 V. Lehmann, J. Electrochem. Soc. , 1993, 140, 2836-2843.

44 P. Granitzer and K. Rumpf, Materials, 2010, 3, 943-998. 